\title{
Association Between Signal Transducers and Activators of Transcription 4 rs7574865 Polymorphism and Systemic Lupus Erythematosus: A Meta-analysis
}

\author{
Young Ho Lee, M.D., Ph.D., Gwan Gyu Song, M.D., Ph.D. \\ Department of Rheumatology, Korea University College of Medicine, Seoul, Korea
}

\begin{abstract}
Objective. The purpose of this study was to determine whether the rs7574865 polymorphism of signal transducers and transcription 4 activators (STAT4) in multiple ethnic populations is associated with susceptibility to systemic lupus erythematosus (SLE). Methods. A meta-analysis on the STAT4 T allele rs7574865 polymorphism was performed in all subjects of study as well as in each ethnic population. Results. It included twenty-four manuscripts with 36 comparative studies of 22,898 SLE patients and 24,838 controls. The mean frequency of the STAT4 rs7574865 T allele was $28.5 \%$, ranging from $14.3 \%$ to $35.7 \%$, among the controls. T allele rates were $14.3 \%, 22.8 \%, 31.9 \%, 32.4 \%$, and 35.7\%, respectively in African American, European, Arab, Asian, and Latin American populations. Meta-analysis revealed a substantial correlation in all subjects between STAT4 rs7574865 and SLE (odds ratio $=1.549,95 \%$ confidence interval $=1.459 \sim 1.644, \mathrm{p}<0.001$ ). Analysis after stratification of race showed a strong association between the STAT4 rs7574865 T allele and SLE in Europeans, Asians, Latin Americans, African Americans, and Arabs. Conclusion. This meta-analysis demonstrated that the STAT4 rs7574865 polymorphism in different ethnic groups was correlated with SLE susceptibility, and that its prevalence depended on ethnicity. (J Rheum Dis 2020;27:277-284)
\end{abstract}

Key Words. STAT4 transcription factor, Genetic polymorphism, Systemic lupus erythematosus, Meta-analysis

\section{INTRODUCTION}

Signal transducers and activators of transcription (STATs) are transcription factors that locate in the cytoplasm, where they are phosphorylated by membranebound receptors and then translocate to the nucleus [1]. STATs control the entire hematopoietic process by regulating cytokine binding to specific cell-surface receptors [1]. STAT4, in particular, is a member of the STAT family, which is activated by interleukin-12 (IL-12) through its receptor and plays an essential downstream role in cell differentiation and proliferation. The system of type I interferon (IFN) plays an important role in the initiation and development of systemic lupus erythematosus (SLE) [2], and STAT4 induces the output of IFN- $\ddot{y}$. STAT4 is also involved in the development of Th17 cells which have a dominant role in autoimmune-associated inflammation [3]. The polymorphism of STAT4 rs7574865 is located within the third intron of the gene. Notably, there is no evidence to suggest that this intronic polymorphism by itself is directly related to changes in protein function. Nevertheless, the STAT4 rs7574865 polymorphism shows the strongest association with autoimmunity, and several SNPs are strongly associated with this intronic SNP [4]. The STAT4 rs7574865 polymorphism has been reported to be associated with SLE in multiple ethnic groups [5-28]. Allelic frequencies of genes are usually significantly different among populations; therefore, ethnicity-specific association studies are needed to confirm genetic associations in various ethnic populations. In this research, we used a meta-analysis method to examine the relationship between the STAT4 rs7574865 polymorphism and SLE

Received : March 31, 2020, Revised : May 8, 2020, Accepted : May 15, 2020

Corresponding to : Young Ho Lee iD http://orcid.org/0000-0003-4213-1909

Division of Rheumatology, Department of Internal Medicine, Korea University Anam Hospital, Korea University College of Medicine, 73 Goryeodae-ro, Seongbuk-gu, Seoul 02841, Korea. E-mail : lyhcgh@korea.ac.kr

Copyright (c) 2020 by The Korean College of Rheumatology.

This is an Open Access article, which permits unrestricted non-commerical use, distribution, and reproduction in any medium, provided the original work is properly cited. 
susceptibility in various populations.

\section{MATERIALS AND METHODS}

\section{Identification of eligible studies and data extraction}

We searched for reports investigating the relationships between SLE and STAT4 polymorphisms. The MEDLINE and EMBASE databases were searched for available studies which analyzed STAT4 polymorphisms in SLE patients (up to January 2020). As Medical Subject Headings (MeSH) or text words, keyword combinations such as "interferon regulatory factor," "STAT4," "polymorphism," "rs7574865," "systemic lupus erythematosus," and "SLE" were entered. References were also investigated in the selected studies to identify additional research not indexed by the electronic databases. No limitations were imposed on language or region. Studies were included if: (1) case-control studies, (2) data were original (independent of studies), and (3) adequate data were given to measure an odds ratio (OR). We excluded studies according to the following criteria: (1) studies containing duplicate data, (2) studies in which they were not possible to determine the number of null and wild genotypes or alleles, and (3) studies in which family members were studied, because these analyzes are based on considerations of linkage. From each research, the following information was extracted: author, year of publication, ethnicity of the study population, demographics, number of cases and controls, and the allele frequency of the STAT4 rs7574865 polymorphism. Any differences among reviewers were resolved by consensus, and the meta-analysis was carried out in accordance with the guidelines of PRISMA [29].

\section{Evaluation of statistical associations}

In each study the frequency of the STAT4 rs7574865 T allele was determined using the method of allele counting. Meta-analyzes were then carried out using the contrast model for the alleles. For each report, point estimates of risk, ORs, and confidence intervals (CI) of $95 \%$ were determined. Moreover, heterogeneities within and between the studies were evaluated using Q-statistics from Cochran. This heterogeneity test evaluated the null hypothesis all studies were evaluating the same effect. The effect of heterogeneity was measured using $\mathrm{I}^{2}$, which ranged from $0 \% \sim 100 \%$ and reflected the proportion of inter-study variation that could be due to heterogeneity rather than chance [30]. Nominally allocated as low mod- erate, and high values were $\mathrm{I}^{2}$ values of $25 \%, 50 \%$, and $75 \%$. The model of fixed effects presumed that genetic factors had similar effects on SLE susceptibility throughout all studies, and that the observed differences between studies were caused by chance alone [31]. The model of random effects assumed that different studies displayed significant variability and measured both in-study sampling error and inter-study variance [32]. In case of significant inter-study heterogeneity, the random effects model was used [33]. Otherwise, fixed effect model was chosen. Statistical manipulations were carried out using the Comprehensive Meta-Analysis software (Biostat, Englewood, NJ, USA).

\section{Evaluation of heterogeneity, sensitivity, and publication bias}

Subgroup analyzes were conducted based on ethnicity in order to explore the potential sources of heterogeneity found in this meta-analysis. In addition, sensitivity analysis was carried out not only to determine the effect of each individual study on pooled ORs by removing each individual study, but also to examine the statistically robust effects of this meta-analysis. While funnel plots have been used to detect publishing bias, they demand several studies of different sample sizes involving subjective judgements. Therefore, we evaluated publishing bias using Egger's linear regression test, which measures funnel plot asymmetry using a natural OR logarithm scale [34].

\section{RESULTS}

\section{Studies included in the meta-analysis}

A total of 259 studies were identified using electronic and manual searches, of which 36 were chosen for a full-text review based on the contents of the title and abstract. Twelve of these 36 studies have been omitted, either because they did not contain data on rs7574865 or overlapping data. Of the 24 studies that met the inclusion criteria [6-29], 3 studies contained data from 3 different groups $[10,24,28], 1$ studies contained data from 5 different groups [22] and 1 studies contained data from 2 different groups [20]. In this meta-analysis, which contained 22,898 SLE patients and 24,838 controls from 11 European, 17 Asian, 4 Latin American, 21 Arab, and 2 African American populations, a total of 36 different comparisons were considered (Table 1). Meta-analyzes specific to ethnicity were carried out on each ethnic population. 
Table 1. Characteristics of individual studies included in meta-analysis

\begin{tabular}{|c|c|c|c|c|c|c|c|c|c|}
\hline \multirow{2}{*}{ Author } & \multirow{2}{*}{ Country } & \multirow{2}{*}{ Ethnicity - } & \multicolumn{2}{|c|}{ Number } & \multicolumn{2}{|c|}{ T allele (\%) } & \multicolumn{3}{|c|}{ Association } \\
\hline & & & SLE & Control & SLE & Control & OR & $95 \% \mathrm{Cl}$ & p-value \\
\hline Gupta et al., 2018 [5] & India & Asian & 394 & 583 & 32.9 & 26.2 & 1.382 & $1.134 \sim 1.685$ & 0.001 \\
\hline Salmaninejad et al., 2017 [6] & Iran & Arab & 50 & 281 & 39.0 & 31.9 & 1.368 & $0.882 \sim 2.123$ & 0.162 \\
\hline Beltrán Ramírez et al., 2016 [7] & Mexico & $\mathrm{LA}$ & 128 & 326 & 53.1 & 39.4 & 1.742 & $1.302 \sim 2.331$ & $<0.001$ \\
\hline Ciccacci et al., 2014 [8] & Italy & European & 239 & 278 & 35.0 & 20.0 & 2.153 & $1.626 \sim 2.849$ & $<0.001$ \\
\hline Dang et al., 2014 [9] & China & Asian & 370 & 576 & 40.1 & 30.9 & 1.499 & $1.236 \sim 1.818$ & $<0.001$ \\
\hline Chai et al.-1, 2014 [10] & Malaysia & Asian & 93 & 110 & 44.1 & 32.7 & 1.621 & $1.082 \sim 2.427$ & 0.019 \\
\hline Chai et al.-2, 2014 [10] & China & Asian & 245 & 294 & 46.3 & 37.8 & 1.423 & $1.115 \sim 1.815$ & 0.005 \\
\hline Chai et al.-3, 2014 [10] & India & Asian & 22 & 26 & 31.8 & 42.3 & 0.636 & $0.275 \sim 1.474$ & 0.291 \\
\hline Kadota et al., 2013 [11] & Japan & Asian & 75 & 190 & 47.3 & & & $1.218 \sim 2$ & 0.003 \\
\hline Mirkazemi et al., 2013 [12] & Iran & Arab & 280 & 281 & 41.3 & 31.9 & 1.502 & $1.176 \sim 1$ & 0.001 \\
\hline Sanchez et al.-1, 2011 [13] & USA & $\mathrm{AF}$ & 1,569 & 1,893 & 16.8 & 14.5 & 1.190 & $1.045 \sim 1$ & 0.009 \\
\hline Sanchez et al.-2, 2011 [13] & USA & $\mathrm{AF}$ & 155 & 131 & 15.0 & 10.7 & 1.493 & $0.906 \sim 2.462$ & 0.116 \\
\hline Li et al., 2011 [14] & China & Asian & 748 & 554 & 41.0 & 43.2 & 0.914 & $0.781 \sim 1.070$ & 0.263 \\
\hline Koga et al., 2011 [15] & Japan & Asian & 282 & 222 & 46.3 & 34.7 & 1.622 & $1.256 \sim 2.096$ & $<0.001$ \\
\hline Sanchez et al., 2010 [16] & USA & $\mathrm{LA}$ & 727 & 595 & 39.1 & 33.9 & 1.247 & $1.063 \sim 1.463$ & 0.007 \\
\hline Kiyohara et al., 2009 [17] & Japan & Asian & 152 & 427 & 37.5 & 29.0 & 1.466 & $1.113 \sim 1.931$ & 0.006 \\
\hline Suarez-Gestal et al., 2009 [18] & Spain & European & 960 & 661 & 33.1 & 24.0 & 1.570 & $1.341 \sim 1.839$ & 0.001 \\
\hline Namjou et al., 2009 [19] & Korea & Asian & 661 & 781 & 33.1 & 24.0 & 1.570 & $1.341 \sim 1.839$ & $<0.001$ \\
\hline al.-1, $2009[20]$ & China & Asian & 910 & 1440 & 46.1 & 33.4 & 1.705 & $1.512 \sim 1.923$ & $<0.001$ \\
\hline I.-2, 2009 [20] & Thai & Asian & 278 & 383 & 48.7 & 35.2 & 1.747 & $1.398 \sim 2$ & $<0.001$ \\
\hline al., 2009 [21] & China & Asian & 8,255 & 4,199 & 42.3 & 32.7 & 1.505 & $1.426 \sim 1.589$ & $<0.001$ \\
\hline et al.-1, 2009 [22] & Germany & European & 247 & 220 & 26.5 & 21.0 & 1.3 & $1.007 \sim$ & 0.045 \\
\hline Abelson et al.-2, 2009 [22] & Italy & European & & 7 & & & & $1.989 \sim 3.695$ & $<0.001$ \\
\hline al.-3, 2009 [22] & Spain & European & 390 & 620 & 32.7 & 22.1 & 1.712 & $1.401 \sim 2.094$ & $<0.001$ \\
\hline t al.-4, $2009[22]$ & Argentina & $\mathrm{LA}$ & & & & & & $1.110 \sim 2.076$ & 0.009 \\
\hline Abelson et al.-5, 2009 [22] & Mexico & $\mathrm{LA}$ & 552 & 633 & 54.7 & 36.4 & 2.109 & $1.789 \sim 2.487$ & $<0.001$ \\
\hline Kawasaki et al., 2008 [23] & Japan & Asian & 308 & 306 & 46.3 & 33.5 & 1.709 & $1.357 \sim 2.153$ & $<0.001$ \\
\hline Kobayashi et al.-1, 2008 [24] & Japan & Asian & 227 & 745 & 43.8 & 31.1 & 1.726 & $1.391 \sim 2.140$ & $<0.001$ \\
\hline Kobayashi et al.-2, 2008 [24] & Japan & Asian & 183 & 938 & 38.5 & 31.0 & 1.397 & $1.107 \sim 1.762$ & 0.005 \\
\hline Kobayashi et al.-3, 2008 [24] & Japan & Asian & 165 & 212 & 43.9 & 30.4 & 1.792 & $1.328 \sim 2.420$ & $<0.001$ \\
\hline Taylor et al., 2008 [25] & USA & European & 1,938 & 2,560 & 31.1 & 22.1 & 1.589 & $1.446 \sim 1.747$ & $<0.001$ \\
\hline Palomino-Morales et al., 2008 [26] & Spain & European & 144 & 410 & 42.7 & 31.5 & 1.624 & $1.232 \sim 2.140$ & 0.001 \\
\hline Harley et al., 2008 [27] & USA & European & 720 & 2337 & 32.0 & 23.0 & 1.576 & $1.384 \sim 1.795$ & $<0.001$ \\
\hline Remmers et al.-1, 2007 [28] & USA & European & 575 & 416 & 31.0 & 22.0 & 1.597 & $1.299 \sim 1.962$ & $<0.001$ \\
\hline Remmers et al.-2, 2007 [28] & USA & European & 349 & 416 & 31.0 & 23.0 & 1.504 & $1.198 \sim 1.889$ & $<0.001$ \\
\hline Remmers et al.-3, 2007 [28] & USA & European & 115 & 416 & 29.0 & 22.0 & 1.453 & $1.046 \sim 2.018$ & 0.026 \\
\hline Total & & & 24,838 & 22,898 & 36.8 & 28.5 & 1.549 & $1.459 \sim 1.644$ & $<0.001$ \\
\hline
\end{tabular}

SLE: systemic lupus erythematosus, OR: odd ratio, Cl: confidence interval, LA: Latin American, AF: African American.

\section{Frequency of the STAT4 rs7574865 T allele in different ethnic groups}

Among the controls, the mean frequency of the STAT4 rs7574865 $\mathrm{T}$ allele was $28.5 \%$, varying from $14.3 \%$ to $35.7 \%$. While the African American population showed the lowest prevalence of $\mathrm{T}$ alleles compared to other ethnic groups, the highest frequency was demonstrated by the Latin American population (Table 2). The T allele frequencies in the African American, European, Arab, Asian, and Latin American populations were 14.3\%, 22.8\%,
$31.9 \%, 32.4 \%$, and $35.7 \%$, respectively, with respect to normal controls.

\section{Association between the STAT4 rs7574865 polymorphism and SLE}

Meta-analysis showed an association in all subjects between SLE and STAT4 rs7574865 polymorphism (OR= $1.549,95 \% \mathrm{CI}=1.459 \sim 1.644, \mathrm{p}<0.001$ ) (Table 3, Figure 1). Analysis after ethnicity stratification revealed that in Europeans, Asians, Latin Americans, African Americans 
Table 2. Prevalence of the STAT4 rs7574865 polymorphism T allele

\begin{tabular}{|c|c|c|c|c|c|}
\hline \multirow{2}{*}{ Population } & \multirow{2}{*}{ No. of studies } & \multicolumn{2}{|c|}{ Numbers } & \multicolumn{2}{|c|}{ T allele (\%) } \\
\hline & & SLE & Control & SLE & Control \\
\hline European & 11 & 5,898 & 8,541 & 32.1 & 22.8 \\
\hline Asian & 17 & 13,368 & 11,986 & 41.6 & 32.4 \\
\hline Latin American & 4 & 1,578 & 1,725 & 45.9 & 35.7 \\
\hline African American & 2 & 1,724 & 2,024 & 12.6 & 14.3 \\
\hline Arab & 2 & 330 & 562 & 40.9 & 31.9 \\
\hline Overall & 36 & 22,898 & 24,838 & 36.8 & 28.5 \\
\hline
\end{tabular}

SLE: systemic lupus erythematosus.

Table 3. Meta-analysis of the STAT4 rs7574865 polymorphism and SLE association

\begin{tabular}{|c|c|c|c|c|c|c|c|c|c|}
\hline \multirow{2}{*}{ Polymorphism } & \multirow{2}{*}{ Population } & \multirow{2}{*}{$\begin{array}{l}\text { No. of } \\
\text { studies }\end{array}$} & \multicolumn{3}{|c|}{ Test of association } & \multicolumn{3}{|c|}{ Test of heterogeneity } & \multirow{2}{*}{$\begin{array}{c}\begin{array}{c}\text { Publication } \\
\text { bias }\end{array} \\
\text { p-value }\end{array}$} \\
\hline & & & OR & $95 \% \mathrm{Cl}$ & p-value & Model & $1^{2}$ & p-value & \\
\hline \multirow[t]{6}{*}{ T allele } & Overall & 36 & 1.549 & $1.459 \sim 1.644$ & $<0.001$ & $\mathrm{R}$ & 69.4 & $<0.001$ & 0.560 \\
\hline & European & 11 & 1.647 & $1.520 \sim 1.785$ & $<0.001$ & $\mathrm{R}$ & 42.4 & 0.066 & 0.323 \\
\hline & Asian & 17 & 1.502 & $1.375 \sim 1.641$ & $<0.001$ & $\mathrm{R}$ & 71.0 & $<0.001$ & 0.920 \\
\hline & Latin American & 4 & 1.624 & $1.223 \sim 2.156$ & 0.001 & $\mathrm{R}$ & 85.4 & $<0.001$ & 0.946 \\
\hline & African American & 2 & 1.207 & $1.064 \sim 1.369$ & 0.003 & $\mathrm{~F}$ & 0 & 0.388 & NA \\
\hline & Arab & 2 & 1.469 & $1.887 \sim 1.819$ & $<0.001$ & $\mathrm{~F}$ & 0 & 0.715 & NA \\
\hline
\end{tabular}

SLE: systemic lupus erythematosus, OR: odd ratio, Cl: confidence interval, F: fixed effect model, R: random effect model, NA: not available.

and Arabs, the STAT4 rs7574866 $\mathrm{T}$ allele was significantly associated with SLE (Table 3, Figure 2).

\section{Association between the STAT4 rs7574865 polymorphism and clinical features of SLE}

STAT4 rs7574865 was more strongly associated with SLE characterized by double-stranded (ds) DNA antibodies, nephritis, and/or age at diagnosis, 30 years $[23,25]$. Each of 3 studies indicated a strong association between the rs7574865 polymorphism and the production of anti-Sm antibodies, pericarditis, hematologic disorders and photosensitivity $[8,14,20]$. However, one study did not detect any correlation between the polymorphism and the specific clinical manifestations of SLE, such as photosensitivity, malar rash, discoid rash, oral ulcer, arthritis, pleuritis, pericarditis, proteinuria, seizures, leucopenia, anti-ds DNA, and antinuclear antibody, although sample size could be the reason for the insignificance in the comparisons [12].

\section{Heterogeneity, sensitivity, and publication bias} Meta-analysis showed between-study heterogeneity between STAT4 rs7574865 polymorphism and SLE in all study subjects and in the populations of Europe, Asia and Latin America; however, no heterogeneity was observed in the population of African America. Sensitivity analysis also showed that no single study significantly affected the pooled OR, and excluding two studies with potential overlap data $[27,28]$ did not significantly affected the pooled OR $(\mathrm{OR}=1.551,95 \% \mathrm{CI}=1.450 \sim 1.658, \mathrm{p}<$ 0.001 ), thereby suggesting that the findings of this meta-analysis are robust. There was no proof of publication bias (Egger's regression test, $\mathrm{p}>0.1$ ) (Table 3, Figure 3 ) in the funnel plot and Egger's regression test.

\section{DISCUSSION}

In this study, through a comprehensive meta-analysis, we updated information regarding the association between the STAT4 rs7574865 polymorphism and SLE. The $\mathrm{T}$ allele prevalence was determined in various populations, and ethnic-specific meta-analyses were performed. Notably, rs7574865 T allele prevalence was found to differ among ethnic populations (14.3\% 35.7\%). The mean $\mathrm{T}$ allele frequency in the controls was $28.5 \%$, which was the lowest among African Americans and the highest 


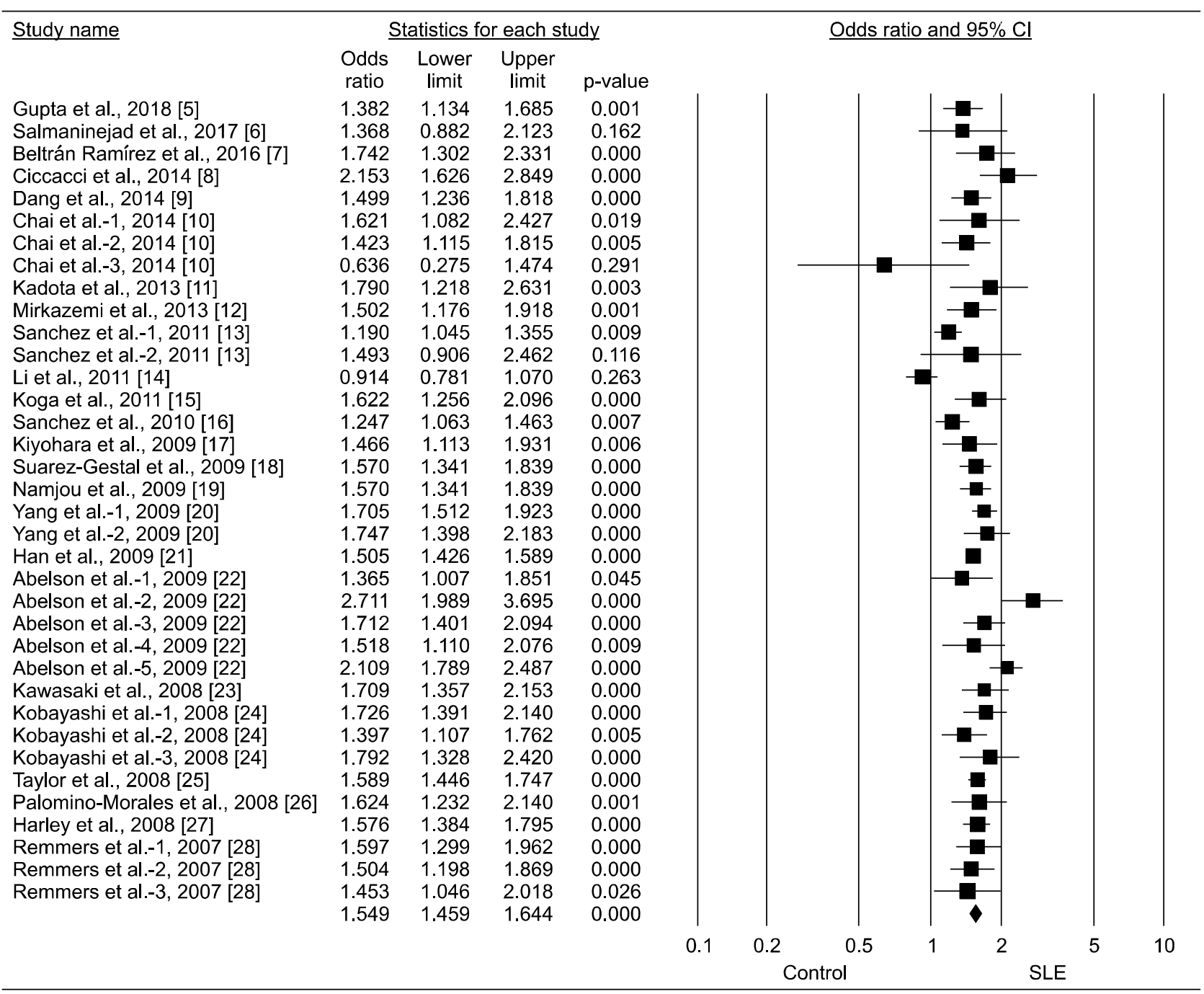

Figure 1. Odd ratio and 95\% confidence interval $(\mathrm{Cl})$ of individual studies and pooled data for the association between the STAT4 rs7574865 polymorphism T allele and systemic lupus erythematosus (SLE) in all subjects.

among Latin Americans. In addition, ethnicity-specific meta-analysis revealed an association among European, Asian, Latin American, African-American Americans, and Arabs.

The result of this meta-analysis suggests that STAT4 could be a genetic risk factor for SLE, showing similar potential across multiple racial groups. Autoimmune diseases are triggered through the breakdown of self-tolerance; therefore, some common pathways are shared. STAT4 is involved in the signaling pathway of several essential cytokines, including IL-12, IFNs, and IL-23 [33]. Previously, STAT4-deficient mice had been shown to be resistant to animal models of diabetes, experimental autoimmune encephalomyelitis, and arthritis [35]. These results suggest that STAT4 could be a common genetic risk factor for autoimmune diseases.
Interestingly, increased expression of type I IFN-regulated genes has previously been documented in the peripheral blood leukocytes of SLE patients, suggesting the role of type I IFN in the pathogenesis of the disease [2]. Notably, rs7574836 polymorphism is located in the third intron of the STAT4 gene, and its functional significance has not yet been established. However, since the haplotype susceptibility is located within STAT4 intron 3, it may be responsible for splice variations or changes in STAT4's regulatory effects. In addition, it has also been shown that the STAT4 risk-haplotype increases the sensitivity of IFN- $\alpha$ signaling [36]. IFN-matured dendritic cells also present an increased risk of autoimmunity by supporting autoimmune T-and B-cells and by generating increased levels of anti-dsDNA antibody, both of which can contribute to the development of SLE [37]. 


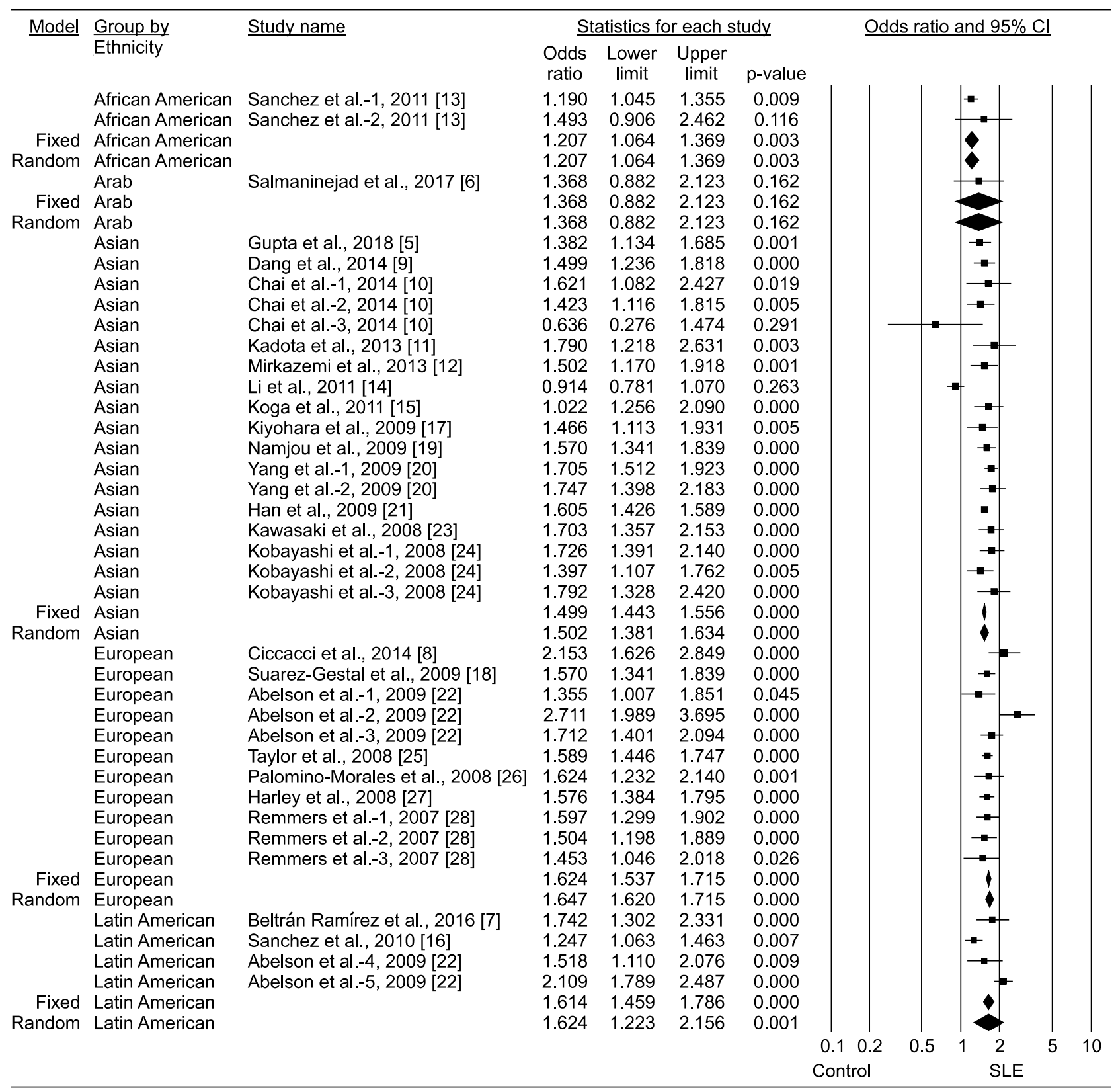

Figure 2. Odd ratios and 95\% confidence intervals (Cls) of individual studies and of pooled data for the association between the T allele and systemic lupus erythematosus (SLE) in each ethnic group.

This meta-analysis differs from an earlier meta-analysis of the relationship between STAT4 rs7574865 polymorphism and SLE risk conducted by Yuan et al. [38], because this study included 16 more studies, 14,445 more SLE patients, and 11,747 more controls in addition to meta-analysis conducted on Latin American and African American populations. The results of this meta-analysis showing the STAT4 rs7574865 polymorphism as a susceptibility factor for SLE in the European and Asian populations are in agreement with the results of the previous study. This meta-analysis also confirmed that the Latin Americans and African Americans associate the STAT4 rs7601754 polymorphism with SLE risk.

There are some limitations to this study which should be considered. First, the analysis may have been affected by heterogeneity and confounding factors, as is commonly found in any other meta-analysis. Second, the STAT4 polymorphism may be associated with clinical manifestations in addition to the susceptibility to disease. However, a thorough meta-analysis was not performed by 


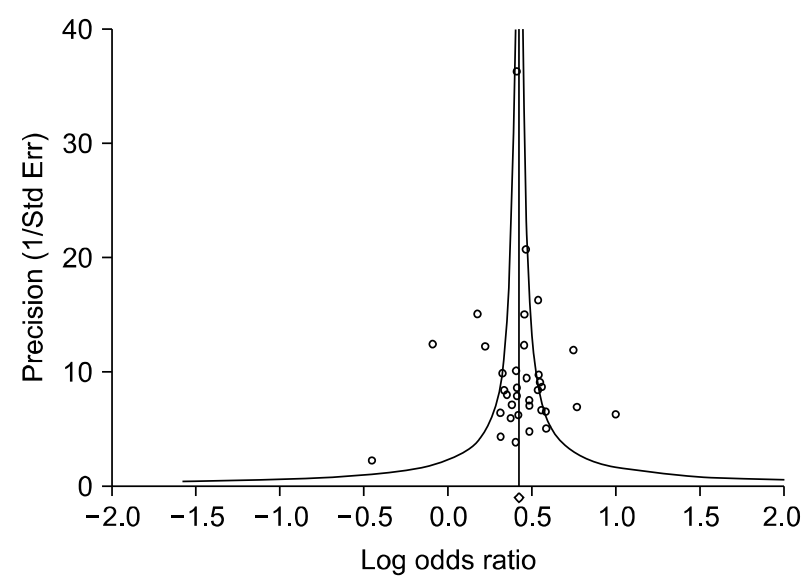

Figure 3. Funnel plot of studies for the association between the $\mathrm{T}$ allele and systemic lupus erythematosus in all subjects (Egger's regression $p$-value $=0.614)$. Std err: standard error.

the limited amount of data available on the association between the STAT4 rs7574865 polymorphism and severity of the disease and/or clinical features.

\section{CONCLUSION}

In conclusion, this meta-analysis, which included 22,898 SLE patients and 24,838 controls, indicates that in multiple ethnic groups the functional STAT4 rs7574865 polymorphism is correlated with SLE susceptibility and that the prevalence of the rs7574865 $\mathrm{T}$ allele depends on ethnicity.

\section{CONFLICT OF INTEREST}

No potential conflict of interest relevant to this article was reported.

\section{AUTHOR CONTRIBUTIONS}

Y.H.L. was involved in conception and design of study, acquisition of data, analysis and/or interpretation of data, drafting the manuscript, revising the manuscript critically for important intellectual content. G.G.S. was involved in conception and design of study, analysis and/or interpretation of data, drafting the manuscript

\section{REFERENCES}

1. Horvath CM. STAT proteins and transcriptional responses to extracellular signals. Trends Biochem Sci 2000;25: 496-502.
2. Banchereau J, Pascual V. Type I interferon in systemic lupus erythematosus and other autoimmune diseases. Immunity 2006;25:383-92.

3. Korman BD, Kastner DL, Gregersen PK, Remmers EF. STAT4: genetics, mechanisms, and implications for autoimmunity. Curr Allergy Asthma Rep 2008;8:398-403.

4. Korman BD, Kastner DL, Gregersen PK, Remmers EF. STAT4: genetics, mechanisms, and implications for autoimmunity. Curr Allergy Asthma Rep 2008;8:398-403.

5. Gupta V, Kumar S, Pratap A, Singh R, Kumari R, Kumar S, et al. Association of ITGAM, TNFSF4, TNFAIP3 and STAT4 gene polymorphisms with risk of systemic lupus erythematosus in a North Indian population. Lupus 2018;27: 1973-9.

6. Salmaninejad A, Mahmoudi M, Aslani S, Poursani S, Ziaee V, Rezaei N. Association of STAT4 gene single nucleotide polymorphisms with Iranian juvenile-onset systemic lupus erythematosus patients. Turk J Pediatr 2017;59:144-9.

7. Beltrán Ramírez O, Mendoza Rincón JF, Barbosa Cobos RE, Alemán Ávila I, Ramírez Bello J. STAT4 confers risk for rheumatoid arthritis and systemic lupus erythematosus in Mexican patients. Immunol Lett 2016;175:40-3.

8. Ciccacci C, Perricone C, Ceccarelli F, Rufini S, Di Fusco D, Alessandri C, et al. A multilocus genetic study in a cohort of Italian SLE patients confirms the association with STAT4 gene and describes a new association with HCP5 gene. PLoS One 2014;9:e111991.

9. Dang J, Shan S, Li J, Zhao H, Xin Q, Liu Y, et al. Gene-gene interactions of IRF5, STAT4, IKZF1 and ETS1 in systemic lupus erythematosus. Tissue Antigens 2014;83:401-8.

10. Chai HC, Chua KH, Lim SK, Phipps ME. Insight into gene polymorphisms involved in toll-like receptor/interferon signalling pathways for systemic lupus erythematosus in South East Asia. J Immunol Res 2014;2014:529167.

11. Kadota K, Mori M, Yanagimachi M, Miyamae T, Hara T, Kanetaka $\mathrm{T}$, et al. Analysis of gender differences in genetic risk: association of TNFAIP3 polymorphism with male childhood-onset systemic lupus erythematosus in the Japanese population. PLoS One 2013;8:e72551.

12. Mirkazemi S, Akbarian M, Jamshidi AR, Mansouri R, Ghoroghi S, Salimi Y, et al. Association of STAT4 rs7574865 with susceptibility to systemic lupus erythematosus in Iranian population. Inflammation 2013;36:1548-52.

13. Sánchez E, Comeau ME, Freedman BI, Kelly JA, Kaufman $\mathrm{KM}$, Langefeld CD, et al. Identification of novel genetic susceptibility loci in African American lupus patients in a candidate gene association study. Arthritis Rheum 2011;63: 3493-501.

14. Li P, Cao C, Luan H, Li C, Hu C, Zhang S, et al. Association of genetic variations in the STAT4 and IRF7/KIAA1542 regions with systemic lupus erythematosus in a Northern Han Chinese population. Hum Immunol 2011;72:249-55.

15. Koga M, Kawasaki A, Ito I, Furuya T, Ohashi J, Kyogoku C, et al. Cumulative association of eight susceptibility genes with systemic lupus erythematosus in a Japanese female population. J Hum Genet 2011;56:503-7.

16. Sanchez E, Webb RD, Rasmussen A, Kelly JA, Riba L, Kaufman KM, et al. Genetically determined Amerindian ancestry correlates with increased frequency of risk alleles for systemic lupus erythematosus. Arthritis Rheum 2010;62: 3722-9. 
17. Kiyohara C, Washio M, Horiuchi T, Tada Y, Asami T, Ide S, et al. Cigarette smoking, STAT4 and TNFRSF1B polymorphisms, and systemic lupus erythematosus in a Japanese population. J Rheumatol 2009;36:2195-203.

18. Suarez-Gestal M, Calaza M, Dieguez-Gonzalez R, PerezPampin E, Pablos JL, Navarro F, et al. Rheumatoid arthritis does not share most of the newly identified systemic lupus erythematosus genetic factors. Arthritis Rheum 2009;60: 2558-64.

19. Namjou B, Sestak AL, Armstrong DL, Zidovetzki R, Kelly JA, Jacob N, et al. High-density genotyping of STAT4 reveals multiple haplotypic associations with systemic lupus erythematosus in different racial groups. Arthritis Rheum 2009; 60:1085-95.

20. Yang W, Ng P, Zhao M, Hirankarn N, Lau CS, Mok CC, et al. Population differences in SLE susceptibility genes: STAT4 and BLK, but not PXK, are associated with systemic lupus erythematosus in Hong Kong Chinese. Genes Immun 2009; 10:219-26.

21. Han JW, Zheng HF, Cui Y, Sun LD, Ye DQ, Hu Z, et al. Genome-wide association study in a Chinese Han population identifies nine new susceptibility loci for systemic lupus erythematosus. Nat Genet 2009;41:1234-7.

22. Abelson AK, Delgado-Vega AM, Kozyrev SV, Sánchez E, Velázquez-Cruz R, Eriksson N, et al. STAT4 associates with systemic lupus erythematosus through two independent effects that correlate with gene expression and act additively with IRF5 to increase risk. Ann Rheum Dis 2009;68: 1746-53.

23. Kawasaki A, Ito I, Hikami K, Ohashi J, Hayashi T, Goto D, et al. Role of STAT4 polymorphisms in systemic lupus erythematosus in a Japanese population: a case-control association study of the STAT1-STAT4 region. Arthritis Res Ther 2008;10:R113.

24. Kobayashi S, Ikari K, Kaneko H, Kochi Y, Yamamoto K, Shimane K, et al. Association of STAT4 with susceptibility to rheumatoid arthritis and systemic lupus erythematosus in the Japanese population. Arthritis Rheum 2008;58: 1940-6.

25. Taylor KE, Remmers EF, Lee AT, Ortmann WA, Plenge RM, Tian C, et al. Specificity of the STAT4 genetic association for severe disease manifestations of systemic lupus erythematosus. PLoS Genet 2008;4:e1000084.

26. Palomino-Morales RJ, Rojas-Villarraga A, González CI, Ramírez G, Anaya JM, Martín J. STAT4 but not TRAF1/C5 variants influence the risk of developing rheumatoid arthritis and systemic lupus erythematosus in Colombians. Genes Immun 2008;9:379-82.

27. International Consortium for Systemic Lupus Erythematosus Genetics (SLEGEN), Harley JB, Alarcón-Riquelme ME, Criswell LA, Jacob CO, Kimberly RP, et al. Genome-wide association scan in women with systemic lupus erythematosus identifies susceptibility variants in ITGAM, PXK, KIAA1542 and other loci. Nat Genet 2008;40:204-10.

28. Remmers EF, Plenge RM, Lee AT, Graham RR, Hom G, Behrens TW, et al. STAT4 and the risk of rheumatoid arthritis and systemic lupus erythematosus. N Engl J Med 2007; 357:977-86.

29. Moher D, Liberati A, Tetzlaff J, Altman DG; PRISMA Group. Preferred reporting items for systematic reviews and metaanalyses: the PRISMA statement. PLoS Med 2009;6: e1000097.

30. Higgins JP, Thompson SG. Quantifying heterogeneity in a meta-analysis. Stat Med 2002;21:1539-58.

31. Egger M, Smith GD, Phillips AN. Meta-analysis: principles and procedures. BMJ 1997;315:1533-7.

32. DerSimonian R, Laird N. Meta-analysis in clinical trials. Control Clin Trials 1986;7:177-88.

33. Watford WT, Hissong BD, Bream JH, Kanno Y, Muul L, O'Shea JJ. Signaling by IL-12 and IL-23 and the immunoregulatory roles of STAT4. Immunol Rev 2004;202:139-56.

34. Egger M, Davey Smith G, Schneider M, Minder C. Bias in meta-analysis detected by a simple, graphical test. BMJ 1997;315:629-34.

35. Chitnis T, Najafian N, Benou C, Salama AD, Grusby MJ, Sayegh MH, et al. Effect of targeted disruption of STAT4 and STAT6 on the induction of experimental autoimmune encephalomyelitis. J Clin Invest 2001;108:739-47.

36. Kariuki SN, Kirou KA, MacDermott EJ, Barillas-Arias L, Crow MK, Niewold TB. Cutting edge: autoimmune disease risk variant of STAT4 confers increased sensitivity to IFNalpha in lupus patients in vivo. J Immunol 2009;182:34-8.

37. Zheng J, Yin J, Huang R, Petersen F, Yu X. Meta-analysis reveals an association of STAT4 polymorphisms with systemic autoimmune disorders and anti-dsDNA antibody. Hum Immunol 2013;74:986-92.

38. Yuan H, Feng JB, Pan HF, Qiu LX, Li LH, Zhang N, et al. A meta-analysis of the association of STAT4 polymorphism with systemic lupus erythematosus. Mod Rheumatol 2010;20:257-62. 